\title{
THE PARKES LENS SURVEY
}

R. L. WEBSTER, P. J. FRANCIS, B. A. HOLMAN, F. J. MASCI

School of Physics,

University of Melbourne,

Parkville, Victoria, 3052, Australia

M. J. DRINKWATER

Anglo-Australian Observatory,

Coonabarabran, NSW, 2357, Australia

AND

B. A. PETERSON

Mount Stromlo and Siding Springs Observatory, Private Bag, Weston Creek, ACT, 2601, Australia

\begin{abstract}
We are undertaking an extensive survey of a sample of radioselected flat-spectrum sources, which are predominantly quasars. The sample will be used for a range of gravitational lensing studies as well as studies of the generic properties of radio quasars. As yet we have not found any instances of multiply-imaged quasars, however we have found evidence for dust which we believe is reddening the observed spectra of many of the quasars. Samples which are selected to be complete in the radio are the best way to account for the effects of dust.
\end{abstract}

\section{Introduction}

Studies of the statistics of gravitational lensing require well-defined samples of source objects, such as quasars. The selection criteria must be specified carefully so that it is possible to determine which manifestations of gravitational lensing might have been detected. In the past, statistical samples have sometimes been chosen from heterogeneous collections of objects such as the Hewett-Burbidge catalogue of quasars. For such studies, it is almost impossible to assess the completeness of the detection limits. 
Types of statistical studies include the numbers of lenses and their distribution with separation, observed magnitude in both optical and radio flux, and image configurations. In future more detailed studies might include other parameters such as variability and spectral characteristics, including evidence of reddening and emission-line strengths. Measurements of the statistics of quasar-galaxy associations require carefully chosen target source lists. For example, closely aligned quasars and galaxies are usually classified as extended galaxies using a simple automated selection procedure. More importantly, sources selected for UV-excess will be biased against quasars behind dusty galaxies. It is very difficult to model the effects of dust, as there is still controversy about the extent and distribution of dust in both spiral and elliptical galaxies. Finally, complete samples of multiply-imaged quasars provide a strong constraint on non-zero values of $\lambda$ as discussed by other contributions in these proceedings.

\section{The Quasar Sample}

We are compiling a complete sample of radio-selected quasars. The sample (Parkes Half-Jansky Flat-Spectrum Sample (PHFS)) comprises 323 sources with fluxes $>0.5 \mathrm{Jy}$ at $2.7 \mathrm{GHz}$. The sources have been chosen to have flat radio spectra: if the radio spectrum from $2.7 \mathrm{GHz}$ to $5.0 \mathrm{GHz}$ is defined by $S(\nu) \propto \nu^{\alpha}$, then sources with $\alpha>-0.5$ are selected. It is well-known that such sources are predominantly quasars, rather than radio galaxies, which are more common amongst the steep-spectrum sources. In addition, the sample was restricted to sources away from the galactic plane, with $|b|>20 \mathrm{deg}$, and with declinations in the range $-45 \mathrm{deg}$ to $+10 \mathrm{deg}$. At present, a total of 258 of the sources have redshifts. Details of the sample will be published shortly (Drinkwater et al. 1996).

Figure 1 shows the redshift distribution of the PHFS compared to a sample of quasars selected by their optical spectra, the Large Bright QSO Survey (Hewett et al. 1995). The two redshift distributions are essentially similar.

Identification of the radio sources has required positions which are accurate to about one arcsecond. These have been obtained using the Australia Telescope Compact Array. The radio positions have then been mapped onto COSMOS maps of the sky. For $92 \%$ of the sources, an optical counterpart is located very close to the radio position; however the remaining sources are fainter than the optical limit of $b_{J} \sim 22.5$ (Drinkwater et al. 1996).

We have conjectured that these sources are reddened, and therefore their $b_{J}$ magnitudes are fainter than the COSMOS limit. We are imaging all sources in the sample in the K-band, and are finding that many of the sources which are faint at optical magnitudes, are many magnitudes 


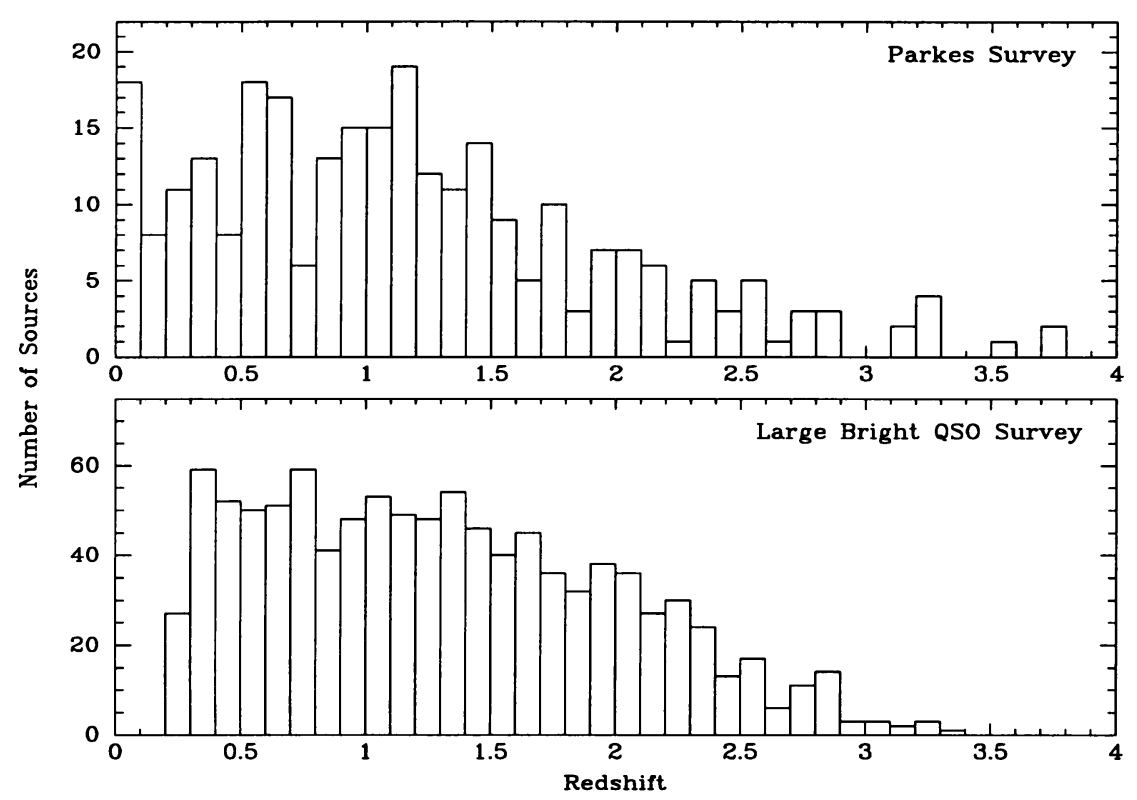

Figure 1. The top histogram shows the distribution of redshifts for the PHFS, and the lower panel shows the redshift histogram for the Large Bright QSO Survey.

brighter in the near-infrared. We measure $b_{J}-K$ colors ranging from $2-$ 10 magnitudes. The redness might either be intrinsic, whereby the source actually has a red continuum, or the source may have been reddened by intervening dust. A strong argument in favor of the latter may be made by considering a plot of emission-line strength against reddening. If the quasar continuum is intrinsically reddened, then an anti-correlation would be predicted between redness and the emission-line strengths. The available data shows no such correlation (Webster et al. 1995).

We are able to compare the sample of radio-selected quasars, with a subsample of QSOs from the Large Bright QSO Survey, for which we have near-infrared data. The latter QSOs, which are largely selected for UVexcess, lie on the blue envelope of the $b_{J}-K$ distribution and have $b_{J}-$ $K$ colors centered around 2.5 magnitudes. Our sample of radio-selected quasars are not biased against dust, and will thus reflect intrinsic quasar colors. Thus we are able to estimate the number of QSOs which would be missed from optically-selected samples, if they have the same reddening distribution as the radio-selected sample. We find that $\sim 80 \%$ of quasars might be missing from these samples (Webster et al. 1995). 


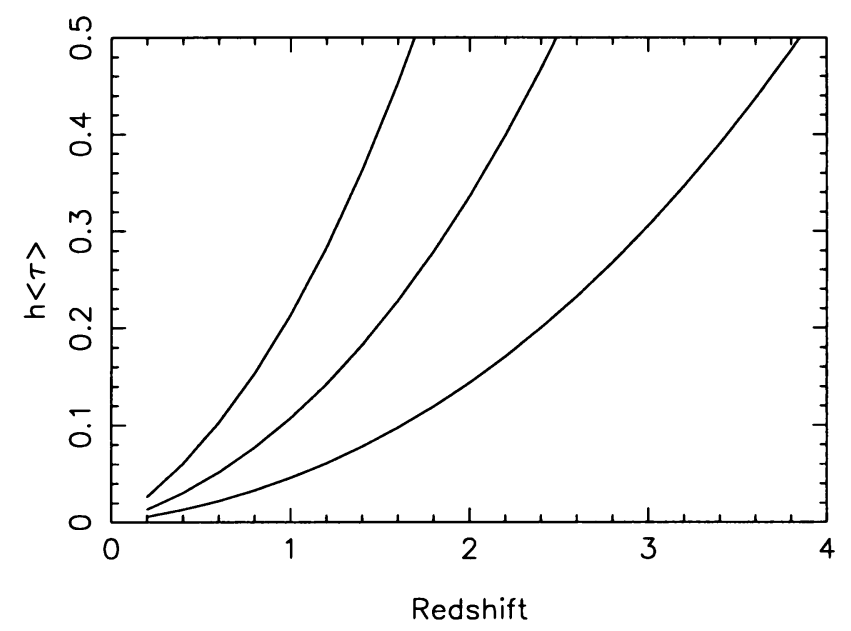

Figure 2. Plot of the mean optical depth in dust (multiplied by the Hubble scaling factor) plotted as function of redshift, for three different galaxy scale lengths.

\section{Dust-Effected Quasars}

There are three possible locations for dust: a line-of-sight galaxy, the presumed host galaxy of the quasar and the quasar environs. We can model the effects of dust in intervening galaxies, and predict the dependence of color on redshift. This effect has been modeled by a number of researchers (see Masci \& Webster (1995) and references therein), who predict a strong dependence with increasing redshift if the dust properties of the galaxies do not evolve with redshift. The actual probability of reddening, which is essentially the optical depth in dust, depends strongly on the assumed parameters for the dust distribution, in particular the spatial extent of the dust.

Generally we expect the lensing galaxies for multiply-images quasars to be strongly biased towards elliptical galaxies, as these have relatively higher velocity dispersions. We have therefore modeled the predicted mean optical depth in dust as a function of redshift for a population of elliptical galaxies. The elliptical galaxies are assumed to have a constant comoving number density of $0.006 \mathrm{Mpc}^{-3}$, and the dust is distributed like the stars, following a Hubble-type law: $\tau(r)=\tau_{B}\left(1+r / r_{0}\right)^{-2}$, where the central optical depth in dust is taken to be $\tau_{B}=2$, and the scale length $r_{0}$ is allowed to vary. Figure 2 shows the mean optical depth in dust as a function of redshift for $r_{0}=10,20,40 \mathrm{kpc}$. For each model the distribution of optical depths in dust is essentially Poissonian, with a substantial tail to high optical depths.

We have constructed a mean quasar spectrum from $1000-12,000 \AA$. 


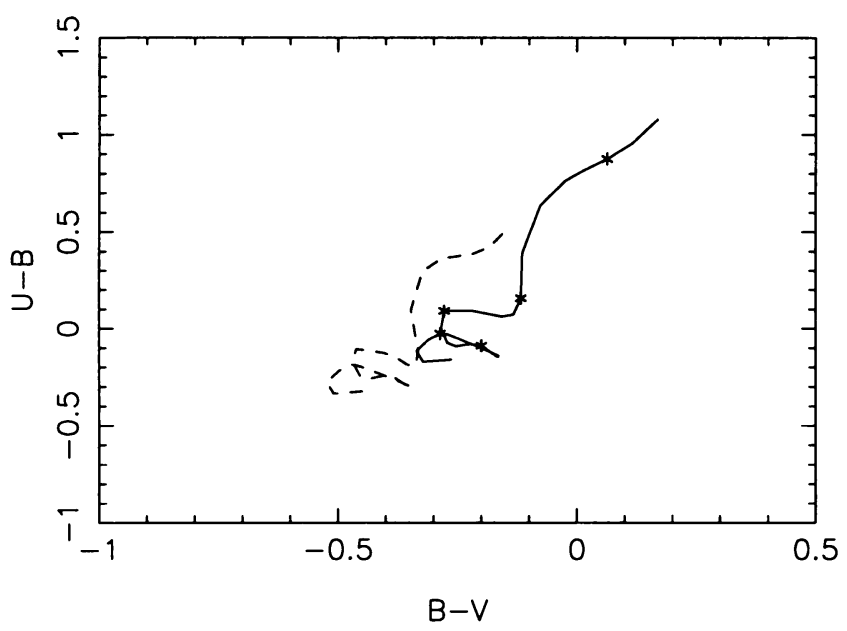

Figure 3. The dotted line shows the two color plot for a quasar with the generic spectrum, over the redshift range $0-2.8$ (higher redshift quasars having more positive colors). The solid line shows the two color plot of the same quasar spectrum reddened at source by $\tau=0.4$ of dust. Redshift intervals of 0.5 are marked. Stars occupy the top left corner of the plot.

The section of the spectrum from $1000-6000 \AA$ is taken from Francis et al. (1991). The red-end of the spectrum has been constructed using a subsample of quasars from the Large Bright QSO Survey; these quasars have been selected principally by their UV-excess, and therefore are not appreciably reddened. The spectrum is a least squares fit to the broadband magnitudes of $\sim 30$ of these quasars (Holman 1996). The generic spectrum is redshifted and convolved with the appropriate filters to construct the two-color plots. The quasar spectrum is then reddened using the mean reddening law derived by (Calzetti et al. 1994).

There are a number of techniques used to separate quasars from stars. In multicolor data, quasars candidates are those sources which lie in the low density parts of the multicolor plots. Thus if the quasars are reddened, and move into the heavily populated stellar locus, they are not detected. Such objects may still be detected by their emission lines.

Figure 3 shows the predicted colors of a generic quasar as a function of redshift. The dashed line is the normal blue quasar. The solid line shows the same quasar spectrum reddened by $\tau=0.4$ in dust located at the same redshift as the source, though the calculation can be readily extended to dust located at intervening redshifts. 


\section{Conclusions}

We have found no candidates gravitational lenses on scales of several arcseconds in our radio-selected sample. However the data is not yet complete enough for us to be able to put strong limits on the lack of observed lensing.

We have found strong evidence for dust-effected quasars. In most cases, it is unlikely that the dust is in the line-of-sight to the quasars. However since the line-of-sight through most galaxies, including ellipticals, will be dusty, these lines-of-sight may be under-represented in QSO samples which are selected by UV-excess techniques. These quasars are also the ones which we expect to be either macro- or microlensed. Thus optically-selected quasar samples may be biased against lensed quasars. Quantification of this bias is difficult. However we note that lists of lensed quasars are over-represented by radio quasars, which are not biased against reddening by dust.

\section{References}

Holman, B.A., 1995, MSc Thesis, University of Melbourne

Calzetti, D., Kinney, A.L. \& Storchi-Bergmann, T., 1994, ApJ, 429, 582

Drinkwater, M.J., Savage, A., Webster, R.L., Condon, J.J., Ellison, S.L., Francis, P.J., Jauncey, D.L., Lovell, J. \& Peterson, B.A., 1996, MNRAS, submitted

Francis, P.J., Hewett,P.C., Foltz, C.B., Chaffee, F.H., Weymann, R.J. \& Morris, S.L., 1991, ApJ, 373, 465

Hewett, P.C., Foltz, C.B. \& Chaffee, F.H., 1995, ApJ, 109, 1498

Masci, F.J. \& Webster, R.L., 1995, Proc Astron Soc Aust, in press

Webster, R.L., Francis, P.J., Peterson, B.A., Drinkwater, M.J. \& Masci, F.J., 1995, Nature, 375,469 